\title{
Pseudo Sigmoid Function Generator for a Superconductive Neural Network
}

\author{
Yuki Yamanashi, Kazumasa Umeda, and Nobuyuki Yoshikawa, Member, IEEE
}

\begin{abstract}
A superconductive perceptron, an artificial neural network, has been investigated using single flux quantum (SFQ) stochastic logic. A superconductive pseudo sigmoid function generator that corresponds to an artificial neuron device for the perceptron has been proposed and implemented using an SFQ current comparator and a frequency-to-current converter, which generates current that is proportional to the average input SFQ frequency. A frequency-to-current converter has been implemented using a dc-SQUID voltage driver coupled with a Josephson transmission line. We implemented and tested the pseudo sigmoid function generator using the SRL $2.5 \mathrm{kA} / \mathrm{cm}^{2} \mathrm{Nb}$ process. The measured input-output characteristic agreed with the ideal sigmoid function with an average error of $0.063 \%$.
\end{abstract}

Index Terms - Artificial neural network, perceptron, comparator, single flux quantum circuit, stochastic logic.

\section{INTRODUCTION}

$\mathrm{B}$ IOLOGICALLY inspired artificial neural networks (ANNs) are thought to be very effective in solving some problems that cannot be efficiently solved by conventional computation systems based on the von Neumann architecture, such as image recognition, pattern recognition, and function approximation. Several devices have been implemented for ANNs using semiconductor devices [1, 2]. However, the operating speeds of such devices are low, and devices that can be used in practical applications have not been built.

In a neural system, signals are represented by voltage pulses. Therefore, a superconductive single flux quantum (SFQ) circuit, which uses single flux quantum pulses as an information carrier [3], is suitable for implementing the ANN in principle. Superconductive ANNs have been proposed and implemented by several research institutes [4-9]. Superconductive stochastic logic, in which data is represented by the density of SFQ pulses in the time domain, has been proposed, and some circuit elements for superconductive ANNs have been implemented [8, 9]. The high-speed SFQ circuit has enables fast operation.

A perceptron, which is a neural network, can approximate arbitrary functions using a superposition of Fourier series expansions, through a learning algorithm [10]. To build hardware for the perceptron, an artificial neuron device, which has a nonlinear input-output characteristic as the activation function, is needed. Among all the nonlinear functions, the

Manuscript received October 9, 2012.

Yuki Yamanashi, Kazumasa Umeda, and Nobuyuki Yoshikawa are with Department of Electrical and Computer Engineering, Yokohama National University, Yokohama 240-8501,Japan. e-mail: yamanasi@ynu.ac.jp sigmoid function is the most suitable mathematically for the learning algorithm. A semiconductor pseudo sigmoid function generator based on sigma-delta modulation was implemented [11]. However, the processing speed was not high because it was necessary to average the output.

In this study, we investigated a superconductive pseudo sigmoid function generator using a superconductive current comparator that has an input-output characteristic that is quite similar to the sigmoid function, on the basis of superconductive stochastic logic. We implemented and tested the pseudo sigmoid function generator.

\section{Perceptron AND Sigmoid FunCtion Generator}

Fig. 1 shows a mathematical model of an artificial neuron. The artificial neuron has multiple inputs and one output. Each input is weighted, and the summation of the weighted inputs is passed through a nonlinear function called an activation function. The operation of the artificial neuron is expressed by the following equations:

$$
\begin{aligned}
& u_{i}[t]=\sum_{j=1}^{N} w_{j} \cdot x_{j}[t] \\
& y[t]=f\left(u_{i}[t]\right),
\end{aligned}
$$

where $u_{i}[t]$ is the sum of the weighted inputs at time $t, w_{j}$ is the weight of the $j$-th input, $x_{j}[t]$ is the $j$-th input, $y[t]$ is the output from the neuron device, and $f$ is an activation function.

Fig. 2 shows the block diagram of a three-layer perceptron, which is composed of input, middle (hidden), and output layers. The perceptron can approximate an arbitrary function using Fourier series by adjusting the weights of the inputs [10]. The accuracy of the approximation improves with an increase in the number of artificial neuron devices in each layer. In the

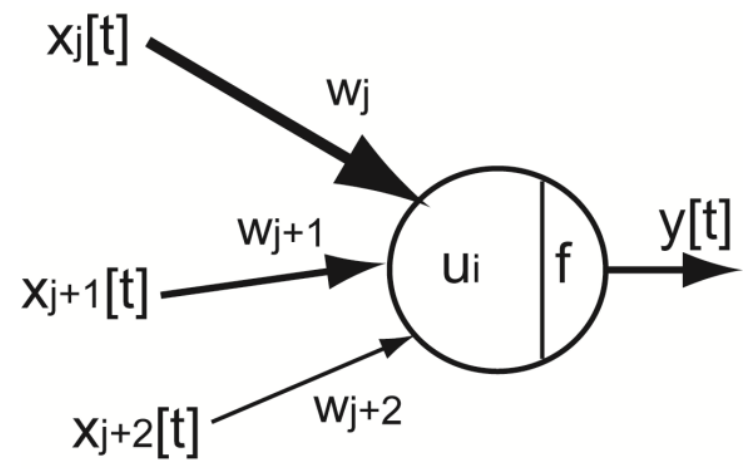

Fig. 1. Mathematical model of artificial neuron. $\mathrm{f}$ is the activation function of the neuron device. A three-input artificial neuron is shown. 


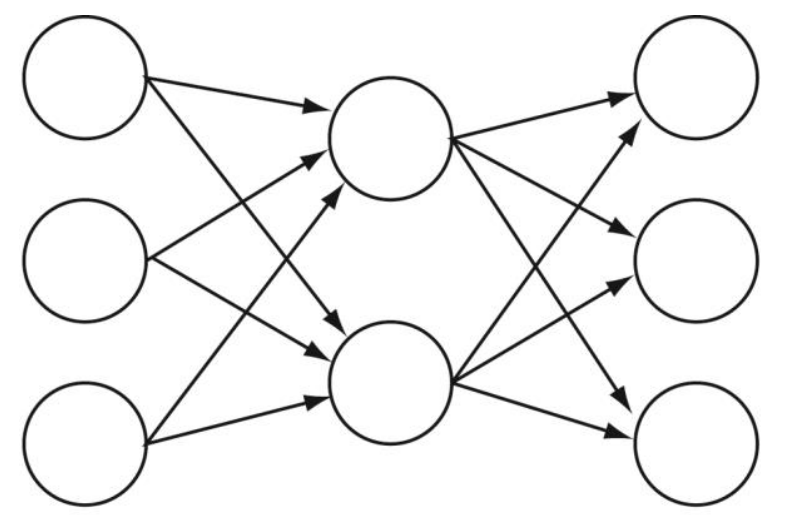

Input layer
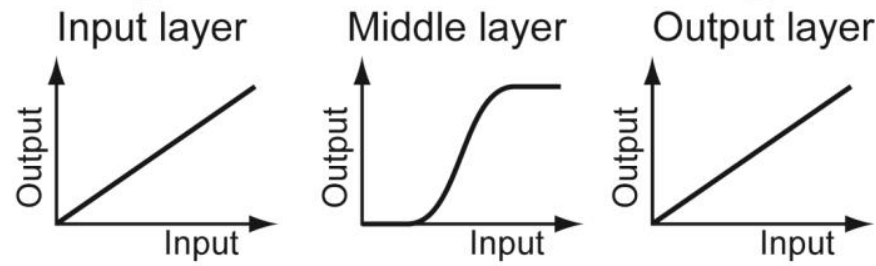

Fig. 2. Structure of artificial neural network, the perceptron. Each circle corresponds to an artificial neuron device. In the middle layer, devices having nonlinear input-output characteristics are required.

input and output layers, linear activation functions are needed as the activation function of the artificial neuron devices. In the middle layer, artificial neuron devices having a nonlinear function are needed for the learning algorithm [10]. A sigmoid function is a nonlinear function and is represented as

$$
f(x)=\frac{1}{1+e^{-a x}}
$$

where $x$ is the input and $a$ is the gain. If we use the sigmoid function as activation functions of the artificial neuron devices in the middle layer, the learning algorithm will converge quickly.

\section{Design OF PSEUdo Sigmoid FunCTION GENERATOR}

In this study, we built a superconductive pseudo sigmoid function using an SFQ comparator. Fig. 3 shows the equivalent circuit of an SFQ comparator. The output probability of the comparator is determined by the value of the current injected into the middle of two Josephson junctions, $\mathrm{J}_{2}$ and $\mathrm{J}_{3}[12,13]$. The SFQ comparator can be used for adjusting the weight of the input for each artificial neuron device. Fig. 4 shows the measured dependence of the output probability of the SFQ comparator shown in Fig. 3. Because of the thermal and quantum noises, the transition in the curve of the output probability versus input current is gradual, as shown in Fig. 4. This transition curve can be fitted by the following sigmoid function,

$$
P\left(I_{i n}\right)=\frac{1}{1+\exp \left\{-1.13\left(I_{i n}-55.0\right)+3.95\right\}}
$$

where $I_{i n}$ is the input current. The average error difference and

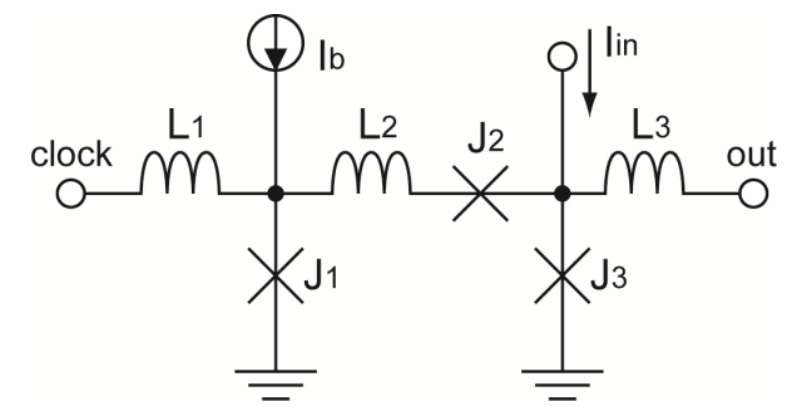

Fig. 3. Equivalent circuit of SFQ comparator. $\mathrm{L}_{1}=\overline{2.5} \mathrm{pH}, \mathrm{L}_{2}=5.0 \mathrm{pH}, \mathrm{J}_{1}=$ $\mathrm{J}_{2}=\mathrm{J}_{3}=216 \mu \mathrm{A}, \mathrm{I}_{\mathrm{b}}=150 \mu \mathrm{A}$.

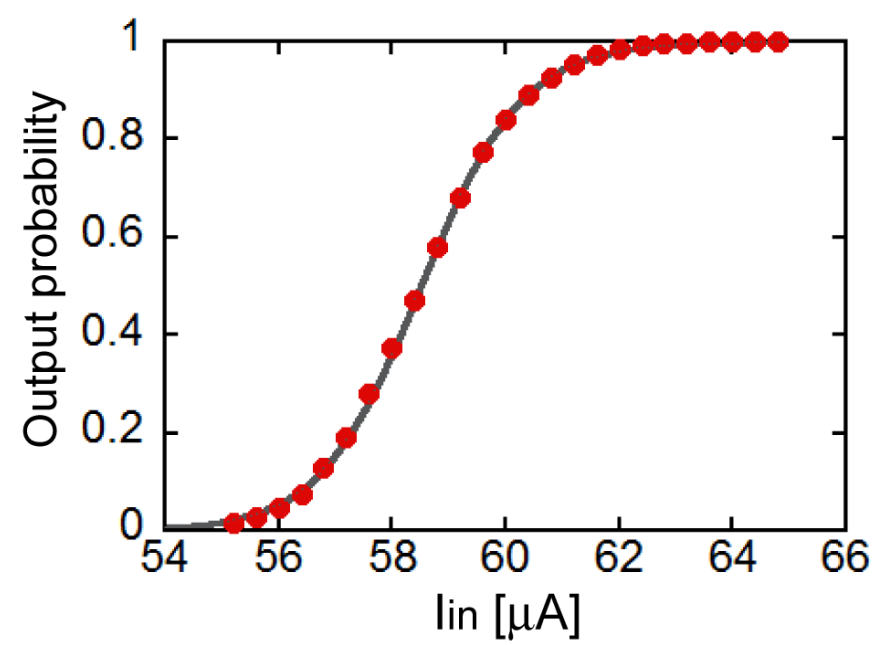

Fig. 4. Measured dependence of output probability on input dc current of SFQ comparator. The dots represent the measurement results. The solid line represents the fitting sigmoid function.

the root mean square error (RMSE) value between the fitted sigmoid function and the measured characteristics are $-0.075 \%$ and $0.62 \%$, respectively. Therefore, the pseudo sigmoid function generator can be implemented using the characteristic of the SFQ comparator. The gain of the sigmoid function and the offset of the input can be adjusted by optimizing the circuit parameters of the SFQ comparator [14].

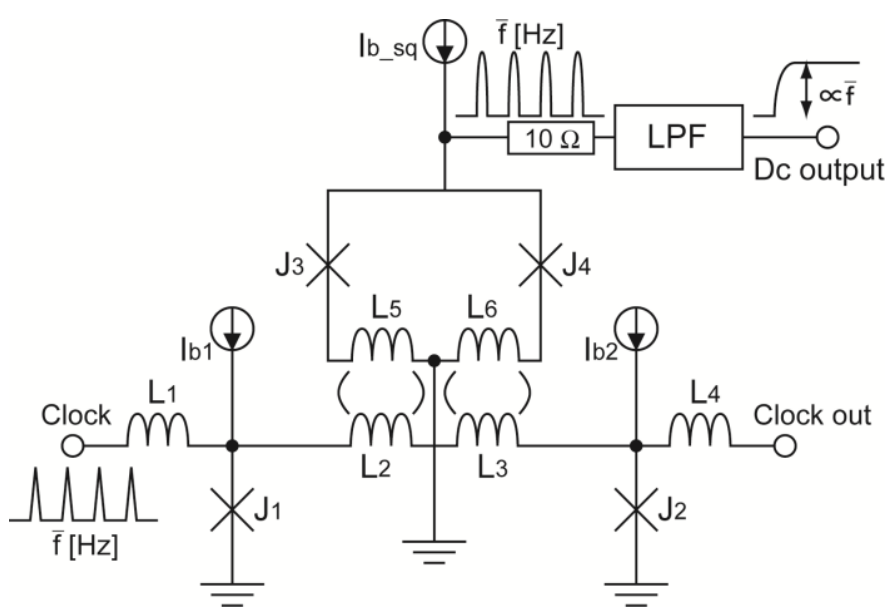

Fig. 5. Equivalent circuit of frequency-to-current comparator based on SFQ stochastic logic. An input SFQ pulse train is converted to dc current by the SQUID voltage generator and the low-pass filter (LPF). $\mathrm{J}_{1}=\mathrm{J}_{2}=216 \mu \mathrm{A}, \mathrm{J}_{3}=$ $\mathrm{J}_{4}=300 \mu \mathrm{A}, \mathrm{L}_{2}=\mathrm{L}_{3}=12.5 \mathrm{pH}, \mathrm{L}_{5}=\mathrm{L}_{6}=14.0 \mathrm{pH}, \mathrm{I}_{\mathrm{b} 1}=\mathrm{I}_{\mathrm{b} 2}=150 \mu \mathrm{A}$. 


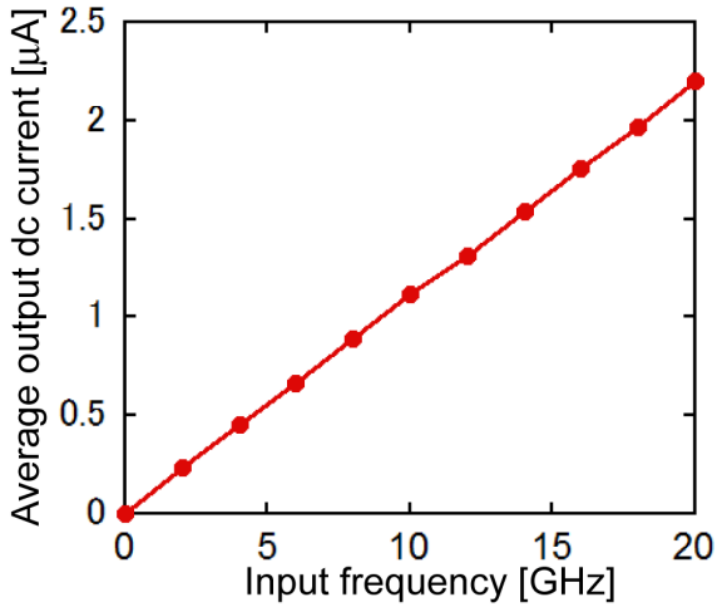

Fig. 6. Dependence of simulated average output current from frequency-tocurrent converter on the SFQ input frequency.

We designed and simulated the superconductive pseudo sigmoid function generator using the SFQ comparator on the basis of SFQ stochastic logic. To generate a current that is proportional to the average SFQ input clock frequency, we devised a frequency-to-current converter. Fig. 5 shows the equivalent circuit of the investigated frequency-to-current converter. A SQUID is biased by $\mathrm{I}_{\mathrm{b}_{-} \mathrm{sq}}$ close to its critical current. An SFQ pulse input to the Josephson transmission line induces a magnetic field to the dc-SQUID, which outputs a voltage pulse. The voltage pulses are generated at the frequency of the SFQ input. By passing the voltage pulses through a resistor and a low-pass filter, a current that is proportional to the SFQ input frequency can be obtained.

Fig. 6 shows a simulated average output current from the frequency-to-current converter as a function of the SFQ input frequency. In this simulation, the cutoff frequency of the lowpass filter was set at $523 \mathrm{MHz}$. In the designed circuit, the low-pass filter of the pseudo sigmoid function generator was a simple LC filter, composed of a $1 \mathrm{pF}$ capacitor and a $1.4 \mathrm{nH}$ inductor, having a cutoff frequency of $523 \mathrm{MHz} .10 \Omega$ resistors were inserted to prevent the perturbation of the operation of the SFQ comparator and the dc-SQUID. As shown in Fig. 6, a linear frequency characteristic was obtained. The fitting linear characteristic is represented as

$$
I_{d c_{-} \text {output }}=0.1091 \bar{f}+0.00115 \text {, }
$$

where $I_{d c_{-} \text {output }}[\mu \mathrm{A}]$ is the output dc current and $\bar{f}[\mathrm{GHz}]$ is the average input clock frequency. The average error between the fitting function and the simulated output current was $1.17 \%$. By combining the investigated frequency-to-current converter and the SFQ comparator, a superconductive pseudo sigmoid function generator has been designed. We verified the correct operation of the pseudo sigmoid function generator through an analog circuit simulation.

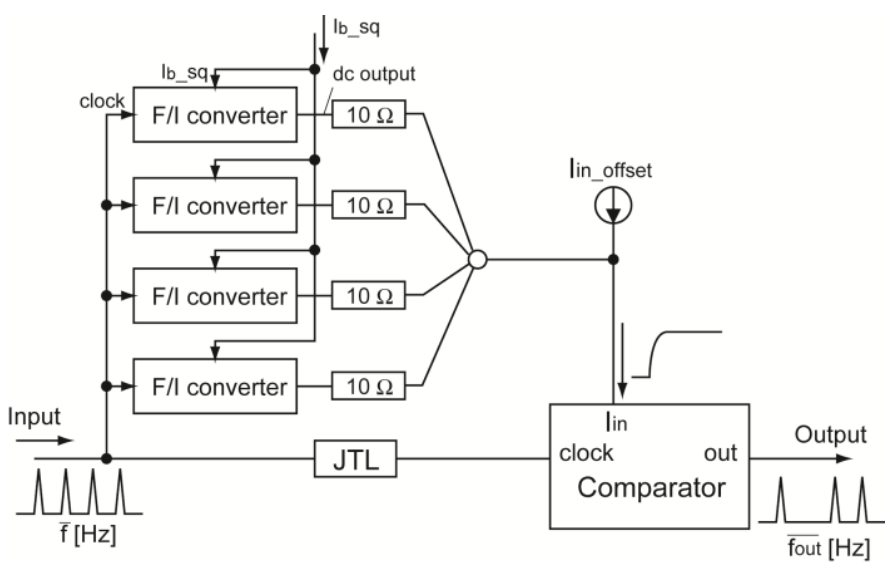

Fig. 7. Block diagram of test circuit of superconductive pseudo sigmoid function generator. The F/I converter corresponds to the frequency-to-current converter. Four frequency-to-current converters placed in parallel were used to supply a large de current to the comparator.

\section{IMPLEMENTATION OF PSEUDO SIGMOID FUNCTION GENERATOR}

We implemented the pseudo sigmoid function generator using the SRL $2.5 \mathrm{kA} / \mathrm{cm}^{2} \mathrm{Nb}$ process [15]. Fig. 7 shows the block diagram of the implemented test circuit of the pseudo sigmoid function generator. To obtain a large current from the input SFQ pulses, four frequency-to-current converters were used. As mentioned in the previous section, a large capacitor was used in the low-pass filter. The use of such a large capacitor requires a large circuit area. The circuit area of the test circuit was $0.9 \mathrm{~mm} \times 1.4 \mathrm{~mm}$. By using a more sophisticated higher order filter and optimizing the circuit parameters, it should be possible to reduce the circuit area. A current input $\mathrm{I}_{\text {in_offset }}$ was supplied to adjust the threshold current of the comparator, where the output probability becomes 0.5 .

The input high-frequency SFQ pulse input was generated by

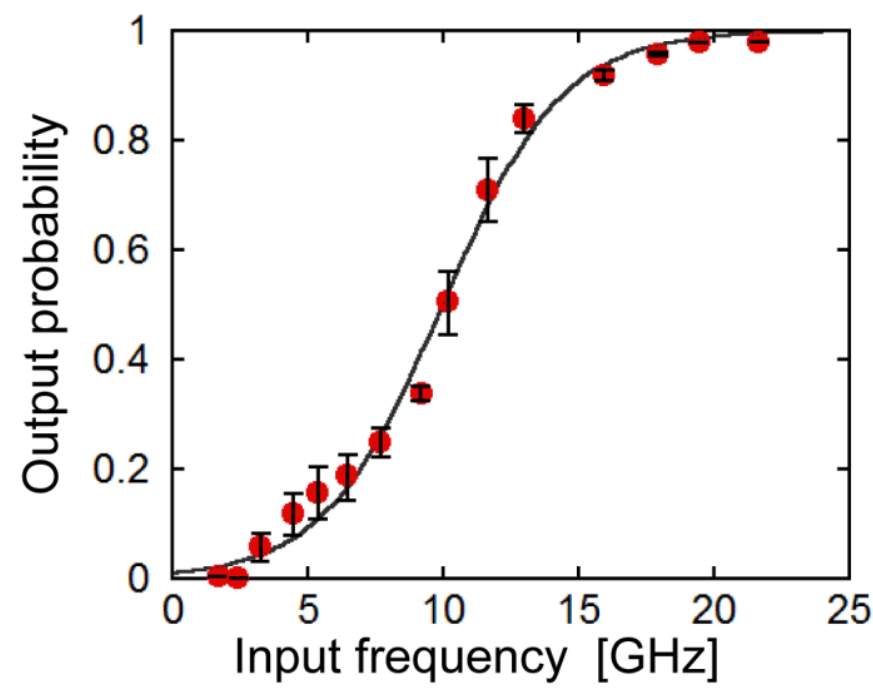

Fig. 8. Measured dependence of output probability of superconductive pseudo sigmoid function generator on input frequency. The dots represent the measurement results. The solid line represents the fitting sigmoid function. 
supplying a current larger than the critical current of the Josephson junction in a Josephson transmission line connected before the pseudo sigmoid function generator. To measure the dependence of the output probability of the pseudo sigmoid function generator on the frequency of the input SFQ pulses, we measured the average input and output voltages of the Josephson junctions corresponding to the average frequencies of the input and output SFQ pulses [16]. During the measurement, the offset current $I_{\text {in_offset }}$ was maintained constant at $35 \mu \mathrm{A}$. By dividing the output average voltage by the input average voltage, the output probability of the pseudo sigmoid function generator was obtained. Because the measured input and output voltages were unstable, we repeated the measurement 1000 times. Fig. 8 shows the measured output probability as a function of the input frequency. The dots represent the measured results, and the solid line represents the fitted sigmoid function, which is represented by

$$
P(f)=\frac{1}{1+\exp (-0.456 f+4.54)} .
$$

The measured error between the fitting function and the measured output probability was $-0.063 \%$. The root mean square error (RMSE) value was $3.33 \%$.

\section{CONCLUSION}

We investigated a superconductive perceptron by using SFQ stochastic logic. We proposed a superconductive pseudo sigmoid function generator using the SFQ comparator. We designed a frequency-to-current converter that outputs a current that is proportional to the average clock input frequency. We implemented the pseudo sigmoid function generator and experimentally verified its correct operation. The measured characteristics agreed with the ideal sigmoid function with an average error of $0.068 \%$.

The next step toward building a practical superconductive ANN is the implementation of a stochastic subtractor, which outputs the difference between two SFQ input frequencies. By using stochastic subtractors and SFQ A/D converters, on-chip control of the weight of each neuron input in the superconductive perceptron becomes feasible because the difference between the outputs from the perceptron and the desired output (teacher data) can be calculated.

\section{ACKNOWLEDGMENT}

This work was supported by "Promotion of Environmental
Improvement for Independence of Young Researchers" under the Special Coordination Funds for Promoting Science and Technology from the Ministry of Education, Culture, Sports, Science and Technology (MEXT) of Japan. The National Institute of Advanced Industrial Science and Technology contributed in part to the circuit fabrication.

\section{REFERENCES}

[1] S. Sato, K. Nemoto, S. Akimoto, M. Kinjo, and K. Nakajima, "Implementation of a new neurochip using stochastic logic," IEEE Trans. Neural Networks, vol. 14, pp. 1122-1127, Sep. 2003.

[2] K. L. Rice, M. A. Bhuiyan, T. M. Taha, C. N. Vutsinas, and M. C. Smith, "FPGA Implementation of Izhikevich Spiking Neural Networks for Character Recognition," Proceedings of the 2009 International Conference on Reconfigurable Computing and FPGAs, pp. 451-456, Dec. 2009.

[3] K. K. Likharev and V. K. Semenov, "RSFQ logic/memory family: A new Josephson-junction technology for sub-terahertz-clock-frequency digital systems," IEEE Trans. Appl. Supercond., vol. 1, pp. 3-28, Mar. 1991

[4] T. Kondo, M. Kobori, T. Onomi, and K. Nakajima, "Design and Implementation of Stochastic Neurosystem Using SFQ Logic Circuits," IEEE Trans. Appl. Supercond., vol. 15, pp. 320-323, Jun. 2005.

[5] T. Hirose, T. Asai, and Y. Amemiya, "Pulsed neural networks consisting of single-flux-quantum spiking neurons," Physica $C$, vol. 463-465, pp. 1072-1075, 2007.

[6] E. D. Rippert and S. Lomatch, “A Multilayered Superconducting Neural Network Implementation," IEEE Trans. Appl. Supercond., vol. 7, pp. 3442-3445, Jun. 1997.

[7] T. Hirose, T. Asai, and Y. Amemiya, "Spiking neuron devices consisting of single-flux-quantum circuits," Physica $C$, vol. 445-448, pp. 10201023,2006

[8] T. Onomi, T. Kondo, and K. Nakajima, "High-speed single fluxquantum up/down counter for neural computation using stochastic logic," J. Phys: Conf. Ser., vol. 97, p.012187, Mar. 2008.

[9] T. Onomi T. Kondo, and K. Nakajima, "Implementation of High-Speed Single Flux-Quantum Up/Down Counter for the Neural Computation Using Stochastic Logic," IEEE Trans. Appl. Supercond., vol. 19, pp. 626-629, Jun. 2009.

[10] G. Cybenko, "Approximation by superpositions of a sigmoid function," Math. Control, Signals Systems, vol. 2, pp. 303-314, 1989.

[11] Y. Murahashi, S. Doki, and S. Okuma, "Realization of Pseudo Sigmoid Function for Pulsed Neuron Based on Delta-Sigma Modulation," IEICE technical report. Neurocomputing, vol. 102, pp.167-172, Mar. 2003.

[12] T. V. Filippov, Y. A. Polyakov, V. K. Semenov, and K. K. Likharev, "Signal resolution of RSFQ comparators," IEEE Trans. Appl. Supercond., vol. 5, pp. 2240-2243, Jun. 1995.

[13] Y. Yamanashi and N. Yoshikawa, "Superconductive Random Number Generator Using Thermal Noises in SFQ Circuits," IEEE Trans. Appl. Supercond., vol. 19, pp. 630-633, Jun. 2009.

[14] B. Ebert, O. Mielke, J. Kunert, R. Stolz, and T. Ortlepp, "Experimentally verified design guidelines for minimizing the gray zone width of Josephson comparators," Supercond. Sci. Technol,. vol. 23, p. 55005, Apr. 2010.

[15] S. Nagasawa, Y. Hashimoto, H. Numata, and S. Tahara, "A 380 ps. 9.5 mW Josephson 4-kbit RAM operated at a high bit yield," IEEE Trans. Appl. Supercond., vol. 5, pp. 2447-2452, Jun. 1995.

[16] S. Iwasaki, M. Tanaka, N. Irie, A. Fujimaki, N. Yoshikawa, H. Terai, and S. Yorozu, "Quantitative evaluation of delay time in the single-fluxquantum circuits," Physica C, vol. 463-465, pp. 1068-1071, May 2007. 